\title{
Corneal surface wettability and tear film stability before and after scleral lens wear
}

\author{
Maria Serramito, Ana Privado-Aroco, Laura Batres, Gonzalo Carracedo G* \\ Department of Optics II (Optometry and Vision), Faculty of Optic and Optometry, Universidad Complutense de Madrid, Madrid, Spain
}

\section{A R T I C L E I N F O}

\section{Keywords:}

Scleral contact lens

Irregular cornea

Corneal videokeratoscope

Tear film surface quality

\begin{abstract}
A B S T R A C T
Purpose: The aim of this study was to evaluate the anterior surface of scleral contact lens and ocular surface wettability before and after one-month of scleral lens wear in patients with keratoconus.

Methods: Forty-nine patients with keratoconus (36.26 \pm 9.03 years) were recruited. The sample was divided into two groups: patients with intrastromal corneal ring (KCICRS group) and patients without ICRS (KC group). TFSQ, Schirmer I test, Ocular Surface Disease Index (OSDI questionnaire), tear break-up time (TBUT) and corneal staining were evaluated in two different visits: Baseline (before lens wear) and one-month visit (10 min after lens removal). Visual Analog Scale (VAS questionnaire) was filled in just after inserting the lenses and just before removing them. TFSQ mean and inferior were evaluated over the contact lens surface at the moment of inserting the lens (baseline visit) and after $8 \mathrm{~h}$ of lens wear (one-month visit).

Results: Anterior corneal surface TFSQ values increased in all groups after scleral lens wear (p $<0.05$ ). However, there were no statistical differences found at the moment of inserting or after $8 \mathrm{~h}$ of lens wear on previous contact lens surface TFSQ $(\mathrm{p}>0.05$ ). No changes were found in tear volume for total and in KC and KCICRS groups ( $\mathrm{p}>0.05$ ). For all groups, there was a statistical decrease of TBUT $(\mathrm{p}<0.05)$. In addition, OSDI score, corneal staining and VAS score improved after scleral lens wear from baseline in total and in both KC and KCICRS groups ( $\mathrm{p}<0.05)$.

Conclusion: The scleral contact lens surface keeps its wettability after one-month of wear. However, the wettability of the ocular surface is worse after contact lens wearing.
\end{abstract}

\section{Introduction}

The tear film is the outermost refractive surface of the eye, and it plays an important role in the quality of vision [1]. Changes in the tear film may cause vision disruption. Contact lenses in the eye are known to alter the structure of the tear film by dividing the pre-corneal tear film into pre-lens and post-lens tear film layers [2,3]. A variety of changes in the quality of the tear film with contact lens wear are noted by unstable tear film, decreased tear film breakup time (TBUT) and increased evaporation and thinning rates [4]. The tear alterations can develop symptoms of dryness and it has been found to be a primary reason for contact lens intolerance. It is also well known that the frequency of dry eye symptoms is higher in contact lens wearers than in non-wearers, with up to $50 \%$ of all contact lens wearers reporting some symptoms of dry eye [5].

Currently, a variety of methods are available to evaluate aspects of the tear film to provide information on its eye surface wettability and stability. The direction of the development has been towards non- invasive techniques, to evaluate a wide area of the ocular surface, and allow the dynamic nature and temporal instability of the tear film to be captured and to be analysed [6]. The new techniques use the local disruption of reflected tear patterns to estimate tear break-up through the high speed videokeratoscopy [7]. This has been used to examine the tear film surface quality (TFSQ) in contact lens wearers and it has demonstrated clear potential for detecting dry eye [8-11]. The high speed videokeratoscopy presents an excellent repeatability [12] and overcomes limitations of some other techniques such as high sensitivity to eye movements with interferometry, errors due to subjective assessment of the tear film with the Tear-Scope and relatively small coverage area with wavefront sensing $[8,13]$.

At present, scleral contact lens can be used as a therapeutic treatment of dry eyes to improve the patient's symptoms of dryness [14-16]. Scleral contact lenses are rigid gas permeable (RGP) with a large diameter designed to rest on the sclera and vault over the entire corneal surface. These lenses have become an important tool in visual rehabilitation of patients with an irregular corneal surface from

\footnotetext{
* Corresponding author at: Faculty of Optic and Optometry, Department Optics II (Optometry and Vision), C/Arcos del Jalon 118, 28032, Madrid, Spain.

E-mail address: jgcarrac@ucm.es (G. Carracedo G).
} 
conditions such as keratoconus and intraestromal corneal rings. The post-lens tear film neutralizes the majority of corneal astigmatism, to correct most of the higher order aberrations and provides the cornea with continuous hydration. Moreover, patients tolerate scleral contact lenses better than corneal RGP lenses due to better comfort, excellent visual acuity and ideal centration [16,17].

The videokeratoscopy has been used to quantify the TFSQ in eyes with and without soft $[8,9,13]$ and rigid [13] contact lens. The studies show that both adversely affect the TFSQ. Nevertheless, there are no studies on the influence of scleral contact lens on the ocular surface nor on contact lens surface wettability, evaluated with the TFSQ. The aim of this study was to evaluate the anterior surface wettability of scleral contact lens and ocular surface wettability before and after one-month of scleral lens wear in patients with irregular corneal surface.

\section{Methods}

\subsection{Patients}

A prospective short-term study was designed, developed, and conducted. Forty-nine (49) subjects with keratoconus were recruited for the study in the Optometry Clinic of the Faculty of Optics and Optometry of the University Complutense of Madrid. Inclusion criterion was keratoconus grade I or II (according to Amsler-Krumeich classification) with or without ICRS. Exclusion criteria were the presence of ocular or lid disease, allergies, and atopy. The sample was divided into 2 groups: 19 subjects with ICRS (KCICRS group), and 30 subjects without ICRS (KC group). The KCICRS group had a ring segment of $120^{\circ}$ to $180^{\circ}$ of arc implanted in the inferior region of the cornea. The study was approved by the Ethics Committee (CEIC) of the Hospital Clínico San Carlos of Madrid. The study was conducted in compliance with good clinical practice guidelines, institutional review board regulations, and the tenets of the Declaration of Helsinki [18]. Subjects signed an informed consent, and they were free to drop out of the research at any time.

All measurements were performed in one eye of each patient, selected randomly. The measurements were performed in two different visits: Baseline visit and one-month visit. Both visits were scheduled between 3-6 pm, avoiding the bias for diurnal variation. One week before the baseline visit, all subjects were fitted with ICD 16.5 (Paragon Vision Sciences, Mesa, AZ) scleral lenses (Paflufocon D material; $\mathrm{Dk}=100$ barrer) with an overall diameter of $16.5 \mathrm{~mm}$ and a centre thickness of 300 microns. Non-preserved saline to fill the lens before application and peroxide cleaner was used daily. All lenses were fitted by the same practitioner. The lens parameters were modified as needed to provide a corneal clearance of 300 to $400 \mu \mathrm{m}$, as suggested by the manufacturer's fitting guide. Corneal clearance was evaluated by slitlamp examination, using an optic section to estimate the tear layer thickness compared with the known lens thickness. Subjects were instructed to wear the scleral lens for $8 \mathrm{~h}$ per day for 1 month. Those who wore scleral contact lenses before the study were required to stop wearing their lenses at least one month before the baseline day.

\subsection{Trials}

TFSQ mean (complete cornea) and TFSQ inferior (corneal inferior area) just before scleral lens wearing (baseline visit) and after $10 \mathrm{~min}$ of lens removal (one-month visit), Schirmer I test, Ocular Surface Disease Index (OSDI questionnaire), tear break-up time (TBUT) and corneal staining was evaluated in both baseline and one-month visit. For Visual Analog Scale (VAS questionnaire), patients had to fill in the questionnaire just after inserting the lenses (baseline visit) and just before removing them (one-month visit). Furthermore, TFSQ mean and TFSQ inferior were evaluated over the anterior contact lens surface on inserting the lens (baseline visit) and after $8 \mathrm{~h}$ of lens wearing (one-month visit).
TFSQ were measured by a corneal topographer (E300; Medmont, Melbourne, Australia) by high-videokeratoscopy system. This technique is based on Placido disk that is reflected from the surface of the tear film on the ocular surface or anterior contact lens. A dynamic corneal topography was performed for $20 \mathrm{~s}$ ( 2 frames per second) after two consecutive blinks, obtaining 40 values of the TFSQ index. Measurements were taken by the same examiner in a laboratory under controlled environmental conditions with an approximate temperature of $24{ }^{\circ} \mathrm{C}$ and humidity of $40 \%$. The reliability and repeatability of this system were described previously [12].

The tear volume was collected using the Schirmer I test [19]. The Schirmer strip was placed on the temporal tarsal conjunctiva of the inferior eyelid for $5 \mathrm{~min}$ with the eyes closed. The test represented tear volume in millimetres. The Ocular Surface Disease Index (OSDI) questionnaire was used to assess dry eye symptomatology and to grade the degree of dry eye symptoms to assist in differentiating dry eye syndrome patients from healthy patients [20]. The questionnaire is composed of 12 questions, each with 5 possible responses with a score between 0 and $4(0$, none of the time; 1 , some of the time; 2 , half of the time; 3 , most of the time; 4 , all of the time). The final score is between 0 and 100 , where 100 corresponds to the highest symptomatology of dry eye.

Visual Analogue Scale was provided to subjectively measure the vision quality and the comfort of the contact scleral lenses. This questionnaire is a $100 \mathrm{~mm}$ long horizontal line where the patients mark the point on the line that best corresponds to their symptom. The final score is between 0 and 100, where 100 points correspond to the best symptomatology and 0 corresponds to the worst symptomatology. After questionnaires, fluorescein was applied to evaluate the tear break-up time (TBUT). A solution was prepared using a $10 \%$ concentration of sodium fluorescein diluted in saline $(\mathrm{NaCl} 0.9 \%)$. For each application, a micropipette with $5 \mu \mathrm{l}$ of diluted fluorescein solution was applied to the inferior conjunctival sac, and $20 \mathrm{~s}$ later, TBUT was analysed using a chronograph to record the time from blink to break in the tear film after the patient was asked to blink twice and keep eyes open. Finally, corneal staining was evaluated with slit lamp by the same examiner. The cornea was divided into five areas to record the grade of staining and, as proposed by the Report of the National Eye Institute and IndustrySponsored Dry Eye Workshop. [21], Cornea and Contact Lens Research Unit (CCLRU) grading scales were used [22].

\subsection{Statistical analysis}

Data were analysed by statistical package SPSS version 23.0 for Windows (SPSS, Inc., Chicago, IL). The values analysed are the means $\pm \mathrm{SD}$ of the experiments performed. For statistical analysis, one eye per subject was selected randomly. Normality of samples was analysed using the Kolgomorov-Smirnov test. Parametric test was used to compare the studied groups. Differences between KC group and KCICRS group were estimated by the Student - $t$-test for independent samples. For intra group comparison, Student t-tests for paired samples were used. $\mathrm{p}<0.05$ was considered statistically significant.

\section{Results}

Demographic characteristics of participants in the study are shown in Table 1. The mean age of the subjects was $36.26 \pm 9.03$ years, with a range of 18 to 62 years. There were statistical differences between KC and KCICRS groups for keratometric data both, flat and steep meridians $(\mathrm{p}<0.05)$, but not for age and sex ( $\mathrm{p}>0.05)$.

All data about TFSQ are summarized in Table 2. The Corneal surface TFSQ mean and inferior area TFSQ values increased after one-month of scleral lens wear in all subjects and in both KC and KCICRS groups, being statistically significant $(\mathrm{p}<0.05)$. However, there were no statistical differences between the moment of inserting and after onemonth of scleral lens wear in TFSQ mean and inferior in the contact lens 
Table 1

Demographic characteristics of participants in the study.

\begin{tabular}{llll}
\hline \multirow{2}{*}{ Parameter } & \multicolumn{2}{l}{ Keratoconus } & \\
\cline { 2 - 4 } & Total & KC group & KCICRS group \\
\hline Patients & 49 & 30 & 19 \\
Mean age (years) \pm SD & $36.26 \pm 9.03$ & $37.70 \pm 7.91$ & $34.00 \pm 10.39$ \\
Age range (years) & {$[18,62]$} & {$[25,62]$} & {$[18,62]$} \\
Gender (male/female) & {$[30,19]$} & {$[18,12]$} & {$[12,7]$} \\
Mean keratometry (D) & & & \\
Flat & $47.23 \pm 4.03$ & $45.71 \pm 3.42$ & $49.47 \pm 3.85$ \\
Steep & $50.60 \pm 4.15$ & $49.27 \pm 4.51$ & $52.55 \pm 4.51$ \\
\hline
\end{tabular}

surface ( $p>0.05$ ). Additionally, these changes are graphically represented in Figs. 1 and 2 (data of these graphs are normalized). Measurements over the cornea surface, Fig. 1 shows a significant increase of TFSQ mean and inferior before contact lens wear in all subjects and in both KC and KCICRS groups. After one-month of scleral lens wear, TFSQ mean increased between 27.41 and $30.47 \%$, and TFSQ inferior between 30.79 and $42.88 \%$ for both KC and KCICRS. In Table 2, it should be noted that TFSQ of the cornea surface for the KCICRS group shows poor tear quality (TFSQ value of 0.30 or greater), worse than the KC group, even before scleral lens wear.

Table 3 shows the tear volume, measured with Schirmer test, break up time (TBUT), Ocular surface index disease (OSDI) score, corneal staining and subjective vision and comfort measured with visual analogue scale (VAS) values before and after scleral lens wear. Figs. 3 and 4 show these differences (data of graph 3 are normalized in percentages). After one-month of scleral lens wear, no changes were found in tear volume in the total group and in KC and KCICRS groups ( $p>0.05$ ). In all groups, there was a statistically significant decrease $(\mathrm{p}<0.05)$ in TBUT without scleral lens after one-month of wearing it. TBUT dropped by $2971 \%$ in the total group, by $2289 \%$ in the KC and by $3897 \%$ in the KCICRS groups (Fig. 3). OSDI score and corneal staining improved statistically significantly in total and in both KC and KCICRS groups $(p<0.05)$. Subjective vision and comfort were better after scleral lens wear than at the moment of inserting them in all subjects and by analyzing KC and KCICRS groups separately $(\mathrm{p}<0.05$ ), showing an improvement in subjective vision of 7,8\% in the KC group and of $6,5 \%$ in the KCICRS group, and an improvement in subjective comfort of $5,4 \%$ in the KC group and of $7,5 \%$ in the KCICRS group (Fig. 4). It should be noted that there were no statistical differences between KC and KCICRS groups in the parameters shown in Table $3(\mathrm{p}>0.05)$.

\section{Discussion}

Nowadays, scleral lenses have been suggested as a treatment for severe dry eye diseases because of their ability to improve the ocular surface integrity [23]. Nevertheless, the presence of a contact lens on

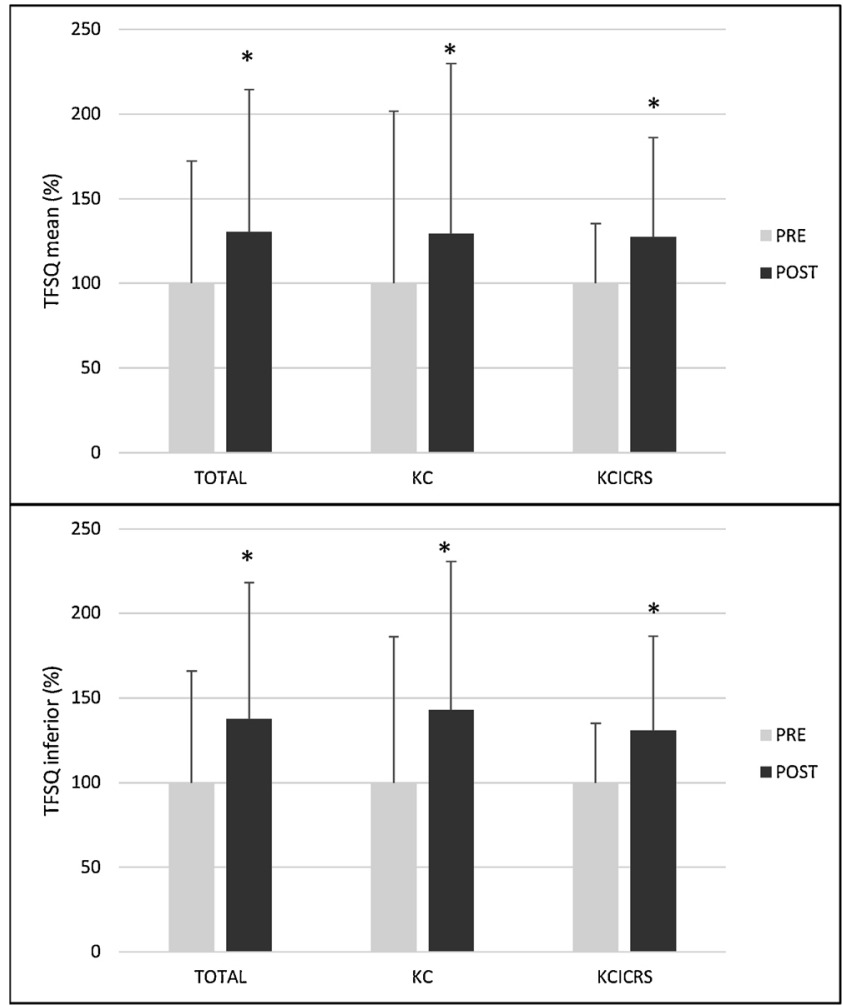

Fig. 1. TFSQ mean and inferior normalized values of anterior corneal surface for total group, KC group and KCICRS group before (Baseline visit) and after one month of scleral contact lens wear (One-month visit). * p value $<0.05$; PRE vs POST; Student $t$-test for related samples.

the eye influences the tear film quality, increases evaporation, and reduces tear film break-up time. TBUT has been described in different contact lens [4,24] and also with scleral contact lens [25,26].

To date, few previous studies have been published in scientific literature about ocular surface wettability before and after wear in patients with irregular corneal surface. Carracedo et al. obtained TBUT slightly lower after wearing a scleral lens for 6-9 hours, but without statistical differences in all groups studied (patients with and without ICRS) $[25,26]$. However, the TBUT found in the present study were lower values and statistically significant, obtaining a reduction of $2.23 \mathrm{~s}$ after contact lens wear. These differences could be due to the patient wearing the contact lens for one month and, in addition, the recruitment of the study was higher. In spite of these outcomes, there are studies that endorse scleral lenses as efficacious and well tolerated for wearing in severe dry eye syndrome $[25,27,28]$.

Another noninvasive method to analyze the tear film is the Tear

Table 2

Tear Film system quality (TFSQ) mean and inferior values before and after scleral lens wear (cornea TFSQ) and on just inserting and previous to removing scleral lenses (CL TFSQ). * $\mathrm{p}$ value $<0.05$. Student's $t$-test for related samples.

\begin{tabular}{|c|c|c|c|c|}
\hline Test & Visit & Total $(n=49)$ & KC group $(n=30)$ & ICRS group $(n=19)$ \\
\hline \multirow[t]{3}{*}{ CL TFSQ mean mean (SD) } & Pre & $0.263 \pm 0.126$ & $0.248 \pm 0.139$ & $0.226 \pm 0.094$ \\
\hline & Post & $0.271 \pm 0.190$ & $0.310 \pm 0.231$ & $0.240 \pm 0.112$ \\
\hline & P-value & 0.730 & 0.997 & 0.705 \\
\hline \multirow[t]{3}{*}{ CL TFSQ inferior mean (SD) } & Pre & $0.290 \pm 0.162$ & $0.310 \pm 0.175$ & $0.255 \pm 0.133$ \\
\hline & Post & $0.301 \pm 0.220$ & $0.322 \pm 0.278$ & $0.272 \pm 0.153$ \\
\hline & P-value & 0.667 & 0.884 & 0.651 \\
\hline \multirow[t]{3}{*}{ Cornea TFSQ mean mean (SD) } & Pre & $0.248 \pm 0.179$ & $0.192 \pm 0.195$ & $0.328 \pm 0.116$ \\
\hline & Post & $0.324 \pm 0.208$ & $0.248 \pm 0.194$ & $0.418 \pm 0.192$ \\
\hline & P-value & $0.005^{*}$ & $0.042^{*}$ & $0.023^{*}$ \\
\hline \multirow[t]{3}{*}{ Cornea TFSQ inferior mean (SD) } & Pre & $0.284 \pm 0.188$ & $0.203 \pm 0.175$ & $0.402 \pm 0.139$ \\
\hline & Post & $0.391 \pm 0.229$ & $0.291 \pm 0.178$ & $0.523 \pm 0.224$ \\
\hline & P-value & $0.031 *$ & $0.028^{*}$ & $0.031^{*}$ \\
\hline
\end{tabular}




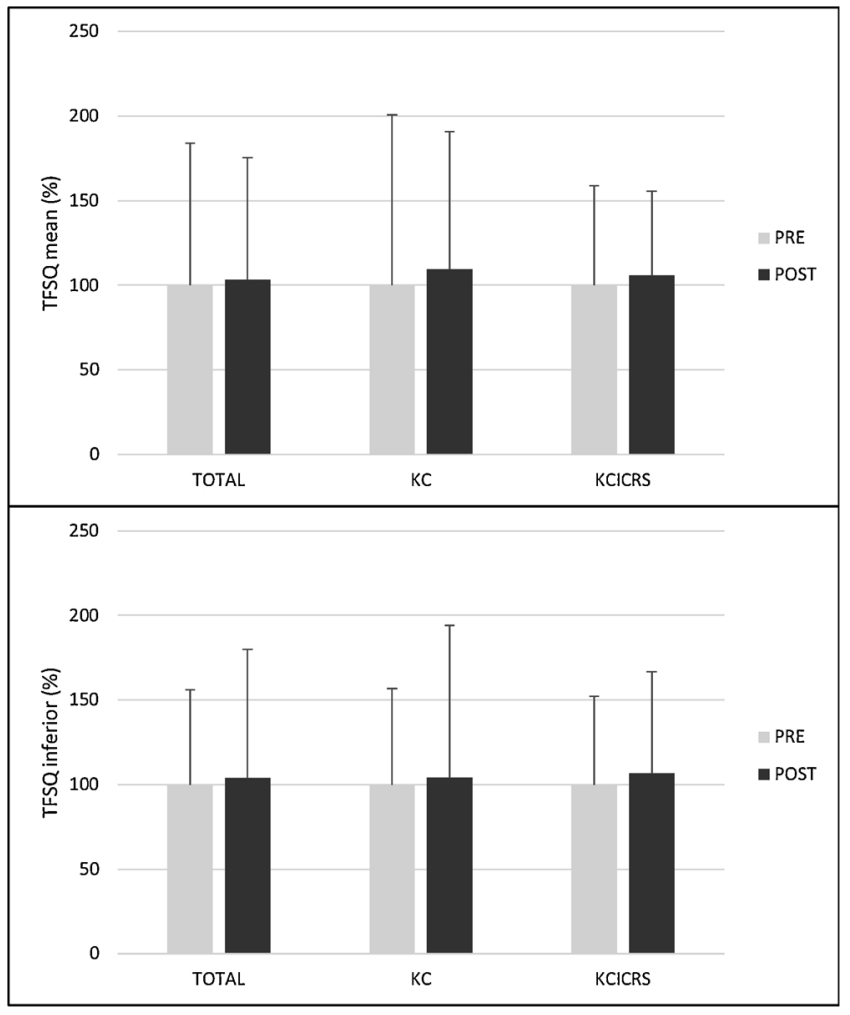

Fig. 2. TFSQ mean and inferior normalized values of anterior contact lens surface for total group, KC group and KCICRS group before (Baseline visit) and after one month of scleral contact lens wear (One-month visit). * p value < 0.05; PRE vs POST; Student $t$-test for related samples.

Film Surface Quality (TFSQ). This technique has been shown to exhibit good precision for tear film measurements, to discriminate dry eye to normal eyes and to assess the quality of the pre-lens tear film with contact lens wear [12]. TFSQ can be measured with different videokeratoscope, such as Keratron 2000 (Optikon, Italy) [29] or E300 corneal topographer (Medmont International Pty Ltd., Victoria, Australia) [30].

In this study, there were no statistical differences in the TFSQ of anterior contact lens surface between baseline (before lens fitting) and after one-month of scleral lens wear, indicating no contact lens

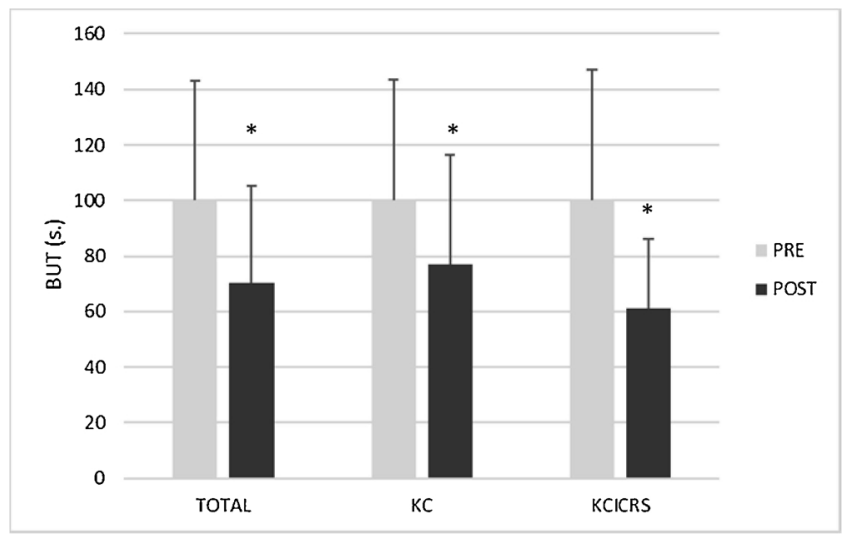

Fig. 3. Tear Break Up Time (BUT) for total group, KC group and KCICRS group before (Baseline visit) and after one month of scleral contact lens wear (Onemonth visit). * $\mathrm{p}$ value $<0.05$; PRE vs POST; Student $t$-test for related samples.

wettability changes. There are publications about TFSQ with soft and rigid corneal lens but not about TFSQ and scleral contact lens. Garima et al. showed that both rigid and soft contact lenses adversely affect the TFSQ in both natural and suppressed blinking conditions but no significant differences were found between the lens types and materials [13]. On the other hand, other authors showed that a material with a higher water gradient improves the TFSQ in contrast to others with a lower water gradient [31]. It could be interesting to perform a longterm study to know if the contact lens material loses its wettability due to deposits and also to assay different maintenance contact lens solutions, different from hydrogen peroxide with moisturizing molecules.

However, TFSQ values of the anterior corneal surface increased after one-month scleral lens wear in both KC and KCICRS groups. After removing the scleral lens, the ocular surface in both groups showed worse wettability. A potential reason to explain this result could be the osmotic difference between the corneal epithelium and saline solution to fill the lens for $8 \mathrm{~h}$ of wear, even inducing a corneal epithelium bogging in some cases, which has been seen to be a sequelae to scleral lens wear [32,33]. More studies to corroborate this statement are mandatory. Furthermore, it should be noted that TFSQ for the KCICRS group without contact lens is worse than the KC group, even before scleral lens wear. This could be due to the corneal surface modification provoked by intrastromal segments implanted in the stroma, molding the anterior surface of the cornea.

Table 3

Tear volume measured with Schirmer test, break up time (BUT), Ocular surface index disease (OSDI) score, corneal staining and subjective vision and comfort measured with visual analogue scale (VAS) values before (baseline visit) and after scleral lens wear (one-month visit). * $p$ value $<0.05$. Student's $t$-test for related samples.

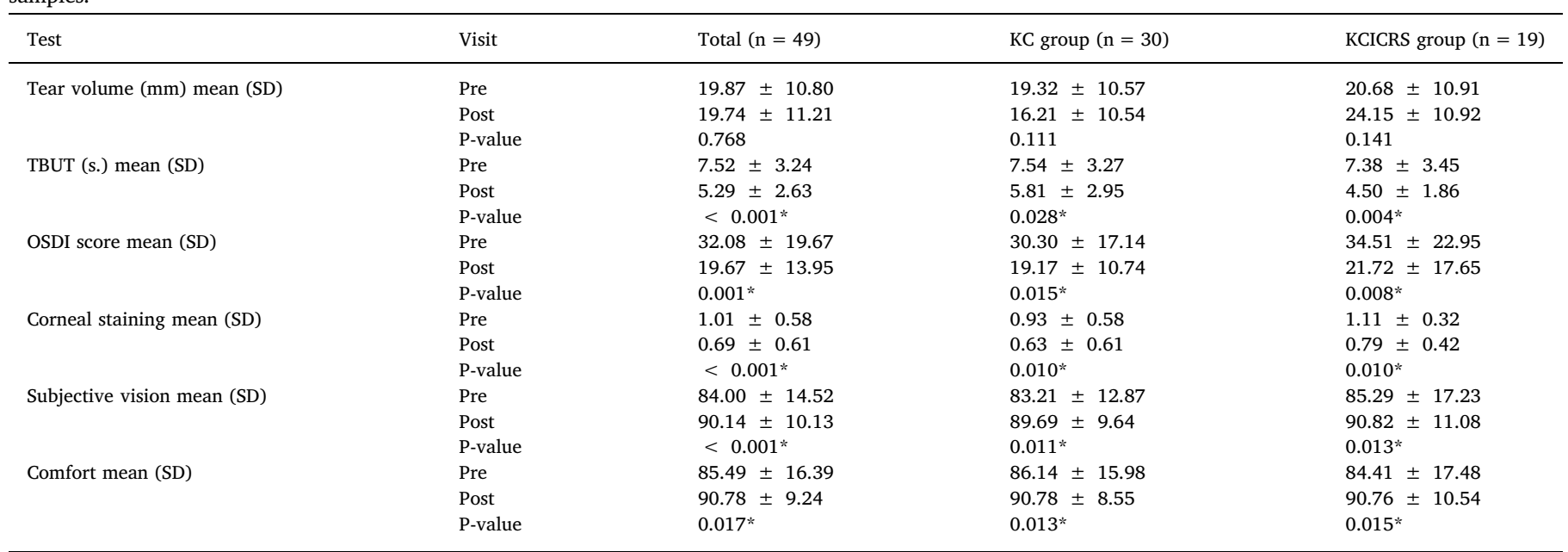




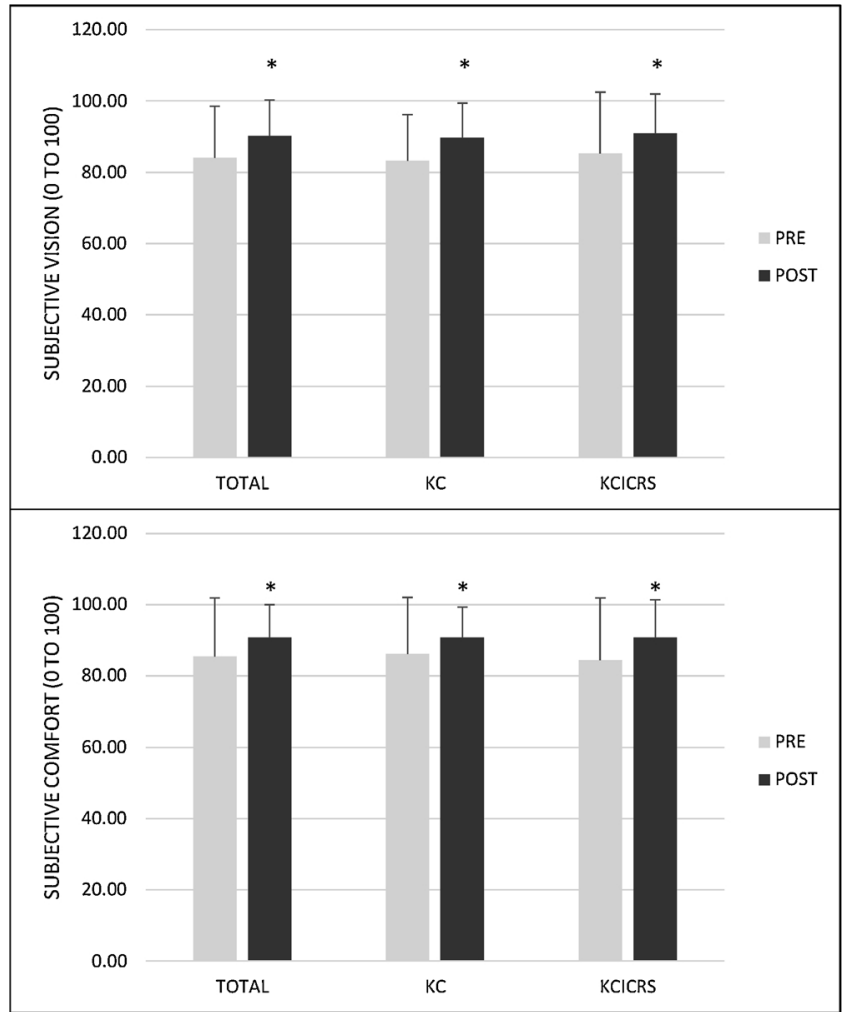

Fig. 4. Subjective vision and comfort, measuring with Visual Analogue Scale (VAS) for total group, KC group and KCICRS group just to insert the contact lens (Baseline visit) and just before removing the lens one month after (One-month visit). * $\mathrm{p}$ value $<0.05$; PRE vs POST; Student $t$-test for related samples.

Ocular surface index disease (OSDI) and corneal staining improved significantly in all the groups. It is well known that scleral contact lens can be used as a therapeutic treatment of dry eyes, improving the patient's symptoms of dryness. An OSDI questionnaire has been used in some previous studies to identify dry eye syndrome in scleral lens wearers [34,35]. Regarding subjective vision and comfort measured with visual analogue scale (VAS), it was found that both were better after scleral lens wear than baseline in both KC and KCICRS groups. These results match well with others described in the scientific literature, confirming one of the most important indications of this kind of contact lens [36-40].

The main limitation of this study is that it is short-term. It might be interesting to evaluate the parameters of this study both 6 months and one year after scleral contact lens wearing to analyze how accumulation of deposits can influence these parameters and also to see the differences between different scleral lens designs, materials, clean and vault solutions. Moreover, this study has been performed in patients with irregular corneas. It could be interesting to assess the same parameters in patients with regular cornea.

In conclusion, short-term scleral lens wearing improves symptomatology of dry eye, vision and comfort. The scleral contact lens surface keeps its wettability after one-month of wear, but the wettability of ocular surface is worse after contact lens wearing.

\section{Disclosure}

The authors do not have any financial interest on the materials and instruments used in this study

\section{References}

[1] R. Montés-Micó, Role of the tear film in the optical quality of the human eye, J
Cataract Refract Surg 33 (9) (2007) 1631-1635.

[2] J. Wang, D. Fonn, T.L. Simpson, L. Jones, Precorneal and pre- and postlens tear film thickness measured indirectly with optical coherence tomography, Invest Ophthalmol Vis Sci 44 (6) (2003) 2524-2528.

[3] J.J. Nichols, P.E. King-Smith, Thickness of the pre- and post-contact lens tear film measured in vivo by interferometry, Invest Ophthalmol Vis Sci 44 (1) (2003) 68-77.

[4] J.J. Nichols, G.L. Mitchell, P.E. King-Smith, Thinning rate of the precorneal and prelens tear films, Invest Ophthalmol Vis Sci 46 (7) (2005) 2353-2361.

[5] M.J. Doughty, D. Fonn, D. Richter, T. Simpson, B. Caffery, K. Gordon, A patient questionnaire approach to estimating the prevalence of dry eye symptoms in patients presenting to optometric practices across Canada, Optom Vis Sci 74 (8) (1997) 624-631.

[6] D.F. Sweeney, T.J. Millar, S.R. Raju, Tear film stability: a review, Exp Eye Res 117 (2013) 28-38.

[7] D.R. Iskander, M.J. Collins, Applications of high-speed videokeratoscopy, Clin Exp Optom 88 (4) (2005) 223-231.

[8] D. Alonso-Caneiro, D.R. Iskander, M.J. Collins, Tear film surface quality with soft contact lenses using dynamic-area high-speed videokeratoscopy, Eye Contact Lens 35 (5) (2009) 227-231.

[9] D.H. Szczesna-Iskander, D.R. Iskander, S.A. Read, D. Alonso-Caneiro, Noninvasive in vivo assessment of soft contact lens type on tear film surface quality, Invest Ophthalmol Vis Sci 53 (1) (2012) 525-531.

[10] D.H. Szczesna, D. Alonso-Caneiro, D.R. Iskander, S.A. Read, M.J. Collins, Predicting dry eye using noninvasive techniques of tear film surface assessment, Invest Ophthalmol Vis Sci 52 (2) (2011) 751-756.

[11] D. Alonso-Caneiro, J. Turuwhenua, D.R. Iskander, M.J. Collins, Diagnosing dry eye with dynamic-area high-speed videokeratoscopy, J Biomed Opt 16 (7) (2011) 076012 .

[12] D.H. Szczesna, D. Alonso-Caneiro, D.R. Iskander, S.A. Read, M.J. Collins, Lateral shearing interferometry, dynamic wavefront sensing, and high-speed videokeratoscopy for noninvasive assessment of tear film surface characteristics: a comparative study, J Biomed Opt 15 (3) (2010) 037005.

[13] G. Tyagi, D. Alonso-Caneiro, M. Collins, S. Read, Tear film surface quality with rigid and soft contact lenses, Eye Contact Lens 38 (3) (2012) 171-178.

[14] J. Sonsino, D.S. Mathe, Central vault in dry eye patients successfully wearing scleral lens, Optom Vis Sci 90 (9) (2013) e248-51 discussion 1030.

[15] D.S. Jacobs, P. Rosenthal, Boston scleral lens prosthetic device for treatment of severe dry eye in chronic graft-versus-host disease, Cornea 26 (10) (2007) 1195-1199.

[16] E. van der Worp, D. Bornman, D.L. Ferreira, M. Faria-Ribeiro, N. Garcia-Porta, J.M. González-Meijome, Modern scleral contact lenses: a review, Cont Lens Anterior Eye 37 (4) (2014) 240-250.

[17] M. Romero-Jiménez, P. Flores-Rodríguez, Utility of a semi-scleral contact lens design in the management of the irregular cornea, Cont Lens Anterior Eye 36 (3) (2013) 146-150.

[18] J.R. Williams, The Declaration of Helsinki and public health, Bull World Health Organ 86 (8) (2008) 650-652.

[19] O.P. van Bijsterveld, Diagnostic tests in the Sicca syndrome, Arch Ophthalmol (Chicago, Ill. : 1960) 82 (1) (1969) 10-14.

[20] R.M. Schiffman, M.D. Christianson, G. Jacobsen, J.D. Hirsch, B.L. Reis, Reliability and validity of the ocular surface disease index, Arch Ophthalmol (Chicago, Ill. 1960) 118 (5) (2000) 615-621.

[21] The definition and classification of dry eye disease: report of the Definition and Classification Subcommittee of the International Dry Eye WorkShop (2007), Ocul Surf 5 (2) (2007) 75-92.

[22] R.L. Terry, C.M. Schnider, B.A. Holden, R. Cornish, T. Grant, D. Sweeney, et al., CCLRU standards for success of daily and extended wear contact lenses, Optom Vis Sci 70 (3) (1993) 234-243.

[23] T. Romero-Rangel, P. Stavrou, J. Cotter, P. Rosenthal, S. Baltatzis, C.S. Foster, Gas permeable scleral contact lens therapy in ocular surface disease, Am J Ophthalmol 130 (1) (2000) 25-32.

[24] L.C. Thai, A. Tomlinson, M.G. Doane, Effect of contact lens materials on tear physiology, Optom Vis Sci 81 (3) (2004) 194-204.

[25] G. Carracedo, M.S. Blanco, A. Martin-Gil, W. Zicheng, J.C. Alvarez, J. Pintor, Shortterm effect of scleral lens on the dry eye biomarkers in Keratoconus, Optom Vis Sci 93 (2) (2016) 150-157.

[26] G. Carracedo, Z. Wang, M. Serramito-Blanco, A. Martin-Gil, J. Carballo-Alvarez, J. Pintor, Ocular surface temperature during scleral lens wearing in patients with keratoconus, Eye Contact Lens (2016).

[27] F. Alipour, A. Kheirkhah, M. Jabarvand Behrouz, Use of mini scleral contact lenses in moderate to severe dry eye, Cont Lens Anterior Eye 35 (6) (2012) 272-276.

[28] J.C. Bavinger, K. DeLoss, S.I. Mian, Scleral lens use in dry eye syndrome, Curr Opin Ophthalmol 26 (4) (2015) 319-324.

[29] D.H. Szczesna-Iskander, D. Alonso-Caneiro, D.R. Iskander, Objective measures of pre-lens tear film dynamics versus visual responses, Optom Vis Sci 93 (8) (2016) 872-880.

[30] D.H. Szczesna-Iskander, Post-blink tear film dynamics in healthy and dry eyes during spontaneous blinking, Ocul Surf 16 (1) (2018) 93-100.

[31] D.H. Szczesna-Iskander, Comparison of tear film surface quality measured in vivo on water gradient silicone hydrogel and hydrogel contact lenses, Eye Contact Lens 40 (1) (2014) 23-27.

[32] M.K. Walker, J.P. Bergmanson, W.L. Miller, J.D. Marsack, L.A. Johnson, Complications and fitting challenges associated with scleral contact lenses: a review, Cont Lens Anterior Eye 39 (2) (2016) 88-96.

[33] S.J. Vincent, D. Alonso-Caneiro, M.J. Collins, The time course and nature of corneal oedema during sealed miniscleral contact lens wear, Cont Lens Anterior Eye (2018). 
[34] L. Magro, J. Gauthier, M. Richet, M. Robin, S. Nguyen, F. Suarez, et al., Scleral lenses for severe chronic GvHD-related keratoconjunctivitis sicca: a retrospective study by the SFGM-TC, Bone Marrow Transplant 52 (6) (2017) 878-882.

[35] J.S. Chahal, M. Heur, G.B. Chiu, Prosthetic replacement of the ocular surface ecosystem scleral Lens therapy for exposure keratopathy, Eye Contact Lens 43 (4) (2017) 240-244.

[36] N. Efron, Obituary-rigid contact lenses, Cont Lens Anterior Eye 33 (5) (2010) 245-252.

[37] D. Fadel, E. Kramer, Potential contraindications to scleral lens wear, Cont Lens
Anterior Eye (2018).

[38] H. Otchere, L. Jones, L. Sorbara, The impact of scleral contact Lens vault on visual acuity and comfort, Eye Contact Lens 44 (Suppl 2) (2018) S54-S59.

[39] P. Yan, M. Kapasi, R. Conlon, J.C. Teichman, S. Yeung, Y. Yang, et al., Patient comfort and visual outcomes of mini-scleral contact lenses, Can J Ophthalmol 52 (1) (2017) 69-73.

[40] E.S. Visser, R. Visser, H.J. van Lier, H.M. Otten, Modern scleral lenses part II: patient satisfaction, Eye Contact Lens 33 (1) (2007) 21-25. 